TAMKANG JOURNAL OF MATHEMATICS

Volume 41, Number 4, 335-347, Winter 2010

\title{
EXISTENCE OF COMMON FIXED POINTS VIA MODIFIED MANN ITERATION IN CONVEX METRIC SPACES AND AN INVARIANT APPROXIMATION RESULT
}

\author{
G. V. R. BABU AND G. N. ALEMAYEHU
}

\begin{abstract}
We prove the existence of common fixed points for two selfmaps $T$ and $f$ of a convex metric space $X$ via the convergence of modified Mann iteration where $T$ is a nonlinear $f$-weakly contractive selfmap of $X$ and range of $f$ is complete. An invariant approximation result is also proved.
\end{abstract}

\section{Introduction}

In 1970, Takahashi [9] introduced a notion of convex metric space and studied the existence of fixed points of nonexpansive maps.

Definition 1.1. (Takahashi [9]). Let $(X, d)$ be a metric space. A mapping $W: X \times$ $X \times[0,1] \rightarrow X$ is said to be a convex structure on $X$ if for each $(x, y, \lambda) \in X \times X \times I$, $I=[0,1]$ and for all $u \in X$,

$$
d(u, W(x, y, \lambda)) \leq \lambda d(u, x)+(1-\lambda) d(u, y) .
$$

A metric space $X$ together with the convex structure $W$ on $X$ is called a convex metric space.

For $x, y \in X, W(x, y, 0)=y$ and $W(x, y, 1)=x$.

Let $X$ be a convex metric space with convex structure $W$. A nonempty subset $M$ of $X$ is said to be convex if $W(x, y, \lambda) \in M$ whenever $(x, y, \lambda) \in X \times X \times I$.

All normed spaces and their convex subsets are convex metric spaces. But there are many examples of convex metric spaces which are not embedded in any normed spaces (Takahashi [9]).

Corresponding author: G. V. R. Babu.

Received June 29, 2009; revised November 11, 2010.

2000 Mathematics Subject Classification. 47H10, 54H25.

Key words and phrases. Weakly compatible maps, modified Mann iteration, common fixed point, invariant approximation. 
As an important extension of the class of contractive mappings, namely the class of weakly contractive mappings was introduced by Alber and Guerre-Delabriere [1] and proved some existence of fixed point results in Hilbert spaces.

In 2001, Rhoades extended this concept to maps defined on Banach spaces as follows:

Definition 1.2. (Rhoades [7]). Let $(X, d)$ be a convex metric space. A mapping $T: X \rightarrow X$ is said to be weakly contractive if

$$
d(T x, T y) \leq d(x, y)-\varphi(d(x, y))
$$

for all $x, y \in X$, where $\varphi: R_{+} \rightarrow R_{+}$, where $R_{+}=[0, \infty)$ satisfying

$$
\begin{aligned}
& \varphi \text { is continuous; } \\
& \varphi \text { is monotone increasing; } \\
& \varphi(0)=0 \\
& \varphi(t)>0 \text { for } t>0
\end{aligned}
$$

and

$$
\lim _{t \rightarrow \infty} \varphi(t)=\infty
$$

It is evident that $T$ is contractive if it is weakly contractive.

Definition 1.3. (Mann [6]). The Mann iteration is defined as follows: For $K$ a convex subset of a Banach space $X$, and $T: K \rightarrow K$, the sequence $\left\{x_{n}\right\}_{n=0}^{\infty}$ in $K$ is defined by

$$
\begin{aligned}
& x_{0} \in K, \\
& x_{n+1}=\left(1-c_{n}\right) x_{n}+c_{n} T x_{n}, n=0,1,2, \ldots,
\end{aligned}
$$

where $\left\{c_{n}\right\}_{n=0}^{\infty}$ satisfies: $0<c_{n} \leq 1$ for all $n$ and $\sum_{n=0}^{\infty} c_{n}=\infty$.

In 2001, Rhoades [7] proved that every weakly contractive map has a unique fixed point in complete metric space and established the following convergence result in Banach spaces.

Theorem 1.4. (Rhoades [7]). Let $K$ be a subset of a Banach space $X$ and $T$ be a weakly contractive selfmap of $K$. Then, for $x_{0} \in K$, the Mann iteration defined by (1.3.1), converges to the unique fixed point $p$ of $T$.

Definition 1.5. (Jungck [4]). Two mappings $A$ and $T$ of a metric space $(X, d)$ are compatible if $\lim _{n \rightarrow \infty} d\left(A T x_{n}, T A x_{n}\right)=0$ whenever $\left\{x_{n}\right\}$ is a sequence in $X$ such that $\lim _{n \rightarrow \infty} A x_{n}=\lim _{n \rightarrow \infty}^{n \rightarrow \infty} T x_{n}=t$ for some $t \in X$. 
Definition 1.6. (Jungck and Rhoades [5]). Two selfmaps $A$ and $T$ of a metric space $(X, d)$ are called weakly compatible if they commute at their coincidence point. That is, for $x \in X$, if $A x=T x$ then $A T x=T A x$.

We observe that every compatible pair of selfmaps is weakly compatible but every weakly compatible pair of selfmaps need not be compatible (Singh and Meade [8]).

Definition 1.7. (Beg and Abbas [3]). Let $(X, d)$ be a metric space. A mapping $T$ : $X \rightarrow X$ is said to be weakly contractive with respect to $f: X \rightarrow X$ if

$$
d(T x, T y) \leq d(f x, f y)-\varphi(d(f x, f y))
$$

for all $x, y \in X$, where $\varphi: R_{+} \rightarrow R_{+}$satisfying (1.2.2), (1.2.3), (1.2.4), (1.2.5) and (1.2.6).

In 2006, Beg and Abbas [3] established the following common fixed point theorem in metric spaces.

Theorem 1.8. (Beg and Abbas [3]). Let $(X, d)$ be a metric space and $T: X \rightarrow X$ be a weakly contractive with respect to $f: X \rightarrow X$. If $T$ and $f$ are weakly compatible, $T(X) \subset f(X)$ and $f(X)$ is a complete subspace of $X$, then $f$ and $T$ have a unique common fixed point in $X$.

Definition 1.9. (modified Mann iteration). Let $(X, d)$ be a convex metric space with convex structure $W$. Let $T$ and $f$ be selfmaps on $X$. Assume that $T(X) \subset f(X)$ and $f(X)$ is a convex subset of $X$. For $x_{0} \in X$, we define a sequence $\left\{f x_{n}\right\}$ in $f(X)$ by

$$
f x_{n+1}=W\left(T x_{n}, f x_{n}, \alpha_{n}\right), n=0,1,2, \ldots,
$$

where $0 \leq \alpha_{n} \leq 1$ for each $n$ and we call the sequence $\left\{f x_{n}\right\}$ a modified Mann iteration.

In 2008, Azam and Shakeel [2] established the convergence of modified Mann iteration in convex metric spaces to a common fixed point of $f$ and $T$, with the help of the existence of the common fixed point that is guaranteed in Theorem 1.8. If Theorem 1.8 is proved in convex metric spaces, then we can apply Theorem 1.8 directly to prove the convergence of modified Mann iteration to the common fixed point of $f$ and $T$. Now the following question is natural.

QUESTION: "Is Theorem 1.8 true in convex metric spaces?"

In this paper we answer this question affirmatively.

Beg and Abbas [3] proved the following convergence theorem in normed linear spaces. 
Theorem 1.10. (Beg and Abbas [3], Theorem 2.6). Let $X$ be a normed space and $T$ and $f$ be selfmaps of $X$. Let $T$ be a weakly contractive mapping with respect to $f$. If $f$ and $T$ are weakly compatible, $T(X) \subset f(X)$ and $f(X)$ is a complete subspace of $X$, then for $x_{0} \in X$, the sequence $\left\{y_{n}\right\}$ in $f(X)$ defined by

$$
y_{n}=f x_{n+1}=\left(1-\alpha_{n}\right) f\left(x_{n}\right)+\alpha_{n} T\left(x_{n}\right), n \geq 0,
$$

where $0<\alpha_{n} \leq 1$ for each $n$ with $\sum_{n=1}^{\infty} \alpha_{n}=\infty$ converges to a common fixed point of $f$ and $T$.

Throughout this paper, we denote $R_{+}=[0, \infty)$ and

$\Phi=\left\{\varphi \mid \varphi: R_{+} \rightarrow R_{+}\right.$satisfies (1.2.2), (1.2.3), (1.2.4), (1.2.5), (1.2.6)

$$
\text { and } \left.t-\varphi(t) \text { is monotonically increasing on } R_{+}\right\} \text {. }
$$

We define $\varphi: R_{+} \rightarrow R_{+}$by $\varphi(t)=\frac{t^{2}}{1+t}, t \geq 0$. Then clearly $\varphi \in \Phi$ so that $\Phi \neq \emptyset$.

The aim of this paper is to prove the existence of common fixed points for two selfmaps $T$ and $f$ of a convex metric space $X$ via the convergence of modified Mann iteration, where $T$ is a nonlinear $f$-weakly contractive selfmap of $X$ and range of $f$ is complete (Theorem 2.1). This theorem answers the question stated above affirmatively. Theorem 1.10 follows as a corollary to Theorem 2.1. In Section 3, an invariant approximation result is proved.

\section{Main results}

The following is the main result of this paper.

Theorem 2.1. Let $(X, d)$ be a convex metric space with convex structure $W$. Let $T, f$ : $X \rightarrow X$ be selfmaps. Assume that there exists a $\varphi \in \Phi$ such that

$$
d(T x, T y) \leq d(f x, f y)-\varphi(d(f x, f y)),
$$

for all $x, y \in X$. If $f(X)$ is complete, then for $x_{0} \in X$, the modified Mann iteration $\left\{f x_{n}\right\}$ defined by (1.9.1) is Cauchy provided $0<\alpha_{n} \leq 1$ for each $n=0,1,2, \ldots$ and $\sum_{n=0}^{\infty} \alpha_{n}=\infty$. Suppose that $\left\{f x_{n}\right\}$ converges to $z$ (say) in $X$. Further, if $T$ and $f$ are weakly compatible, then $z$ is the unique common fixed point of $f$ and $T$.

Proof. For each positive integer $n$, define

$$
\begin{aligned}
A_{n} & =\left\{f x_{i}\right\}_{i=0}^{n} \cup\left\{T x_{i}\right\}_{i=0}^{n} . \\
\text { Write } a_{n} & =\operatorname{diam}\left(A_{n}\right) .
\end{aligned}
$$

If $a_{n}=0$ for some $n$, then $f x_{i}=T x_{j}$ for all $0 \leq i, j \leq n$ and hence $f x_{0}=T x_{0}=z$ (say). 
Since the pair of mappings $(T, f)$ is weakly compatible, $f T x_{0}=T f x_{0}$ and hence $f z=T z$.

We now claim that $T z=z$. Suppose $T z \neq z$.

Consider

$$
\begin{aligned}
d(T z, z)=d\left(T z, T x_{0}\right) & \leq d\left(f z, f x_{0}\right)-\varphi\left(d\left(f z, f x_{0}\right)\right) \\
& =d(T z, z)-\varphi(d(T z, z)),
\end{aligned}
$$

a contradiction. Hence, $T z=z$.

Hence, $f z=T z=z$.

Since $f x_{n}=f x_{0}$ for all $n$, we have $\lim _{n \rightarrow \infty} f x_{n}=f x_{0}=z$.

Hence, without loss of generality, we assume $a_{n}>0$ for all $n$.

$$
\text { Write } b_{n}=\operatorname{Sup}\left\{d\left(f x_{0}, T x_{i}\right): 0 \leq i \leq n\right\} \text {. }
$$

We claim that $a_{n}=b_{n}$ for all $n$.

Clearly $b_{n} \leq a_{n}$ for all $n$. To show that $a_{n} \leq b_{n}$, we consider the following three cases.

Case (i). $a_{n}=d\left(T x_{i}, T x_{j}\right)$ for some $0 \leq i, j \leq n$.

Now,

$$
\begin{aligned}
a_{n} & =d\left(T x_{i}, T x_{j}\right) \leq d\left(f x_{i}, f x_{j}\right)-\varphi\left(d\left(f x_{i}, f x_{j}\right)\right) \\
& \leq a_{n}-\varphi\left(a_{n}\right),
\end{aligned}
$$

a contradiction.

Hence, $a_{n} \neq d\left(T x_{i}, T x_{j}\right)$ for all $0 \leq i, j \leq n$.

Case (ii). $a_{n}=d\left(f x_{i}, T x_{j}\right)$ for some $0 \leq i, j \leq n$.

If $i=0$, then $a_{n}=d\left(f x_{0}, T x_{j}\right) \leq b_{n}$ for all $n \geq 0$.

If $i \neq 0$, then

$$
\begin{aligned}
a_{n}=d\left(f x_{i}, T x_{j}\right) & =d\left(W\left(T x_{i-1}, f x_{i-1}, \alpha_{i-1}\right), T x_{j}\right) \\
& \leq \alpha_{i-1} d\left(T x_{i-1}, T x_{j}\right)+\left(1-\alpha_{i-1}\right) d\left(f x_{i-1}, T x_{j}\right) .
\end{aligned}
$$

If $\alpha_{i-1}=1$, we get $a_{n}=d\left(T x_{i-1}, T x_{j}\right)$ which does not hold by case (i).

If $\alpha_{i-1} \in(0,1)$, from $(2.1 .5)$,

$$
a_{n} \leq \alpha_{i-1} a_{n}+\left(1-\alpha_{i-1}\right) d\left(f x_{i-1}, T x_{j}\right)
$$


which implies that

$$
a_{n} \leq d\left(f x_{i-1}, T x_{j}\right) \leq a_{n}
$$

and hence $a_{n}=d\left(f x_{i-1}, T x_{j}\right)$.

Repeating this procedure, we obtain $a_{n}=d\left(f x_{0}, T x_{j}\right) \leq b_{n}$ for all $n \geq 0$.

Hence, $a_{n} \leq b_{n}$ for all $n \geq 0$.

Case (iii). $a_{n}=d\left(f x_{i}, f x_{j}\right)$ for some $0 \leq i<j \leq n$.

Now,

$$
\begin{aligned}
a_{n}=d\left(f x_{i}, f x_{j}\right) & =d\left(f x_{i}, W\left(T x_{j-1}, f x_{j-1}, \alpha_{j-1}\right)\right) \\
& \leq \alpha_{j-1} d\left(f x_{i}, T x_{j-1}\right)+\left(1-\alpha_{j-1}\right) d\left(f x_{i}, f x_{j-1}\right) .
\end{aligned}
$$

If $\alpha_{i-1}=1$, we get $a_{n}=d\left(f x_{i}, T x_{j-1}\right)$ which is discussed in case (ii).

If $\alpha_{i-1} \in(0,1)$, we get $a_{n}=d\left(f x_{i}, f x_{j-1}\right)$.

Repeating this procedure, we obtain $a_{n}=d\left(f x_{i}, T x_{i}\right)=0$, a contradiction.

Hence from all the three cases, we have $a_{n} \leq b_{n}$ for all $n$.

Thus, $a_{n}=b_{n}$ for all $n$.

Next we claim that the sequences $\left\{f x_{n}\right\}$ and $\left\{T x_{n}\right\}$ are bounded. For this purpose, we show that $\left\{a_{n}\right\}$ is bounded.

Suppose that $\left\{a_{n}\right\}$ is not bounded. Then there exists a subsequence $\left\{a_{n_{k}}\right\}$ of $\left\{a_{n}\right\}$ such that $\lim _{k \rightarrow \infty} a_{n_{k}}=\infty$.

Then, for $0 \leq k \leq n_{k}$, we have

$$
\begin{aligned}
d\left(f x_{0}, T x_{k}\right) & \leq d\left(f x_{0}, T x_{0}\right)+d\left(T x_{0}, T x_{k}\right) \\
& \leq \gamma+d\left(f x_{0}, f x_{k}\right)-\varphi\left(d\left(f x_{0}, f x_{k}\right)\right) \\
& \leq \gamma+a_{n_{k}}-\varphi\left(a_{n_{k}}\right)
\end{aligned}
$$

so that

$$
b_{n_{k}}=\operatorname{Sup}\left\{d\left(f x_{0}, T x_{k}\right): 0 \leq k \leq n_{k}\right\} \leq \gamma+a_{n_{k}}-\varphi\left(a_{n_{k}}\right),
$$

and hence

$$
a_{n_{k}} \leq \gamma+a_{n_{k}}-\varphi\left(a_{n_{k}}\right) \text {, where } \gamma=d\left(f x_{0}, T x_{0}\right) .
$$

This shows that $\varphi\left(a_{n_{k}}\right) \leq \gamma$

and hence

$$
\lim _{k \rightarrow \infty} \varphi\left(a_{n_{k}}\right) \leq \gamma,
$$

a contradiction, since $\lim _{k \rightarrow \infty} \varphi\left(a_{n_{k}}\right)=\infty$. 
Hence, $\left\{a_{n}\right\}$ is bounded.

Next we claim that $\left\{f x_{n}\right\}$ is Cauchy.

Write $E_{n}=\left\{f x_{i}\right\}_{i \geq n} \cup\left\{T x_{i}\right\}_{i \geq n}$ and

$$
e_{n}=\operatorname{diam}\left(E_{n}\right) .
$$

Now write

$$
\beta_{n}=\operatorname{Sup}\left\{d\left(f x_{n}, T x_{j}\right): j \geq n\right\} \text { for each } n .
$$

We claim that $e_{n}=\beta_{n}$ for all $n$.

Clearly $\beta_{n} \leq e_{n}$ for all $n$.

We now show that $e_{n} \leq \beta_{n}$ for all $n$.

Case (i). $e_{n}=d\left(T x_{i}, T x_{j}\right)$ for some $i, j \geq n$.

Now,

$$
\begin{aligned}
e_{n} & =d\left(T x_{i}, T x_{j}\right) \leq d\left(f x_{i}, f x_{j}\right)-\varphi\left(d\left(f x_{i}, f x_{j}\right)\right) \\
& \leq e_{n}-\varphi\left(e_{n}\right),
\end{aligned}
$$

a contradiction.

Hence, $e_{n} \neq d\left(T x_{i}, T x_{j}\right)$ for all $i, j \geq n$.

Case (ii). $e_{n}=d\left(f x_{i}, T x_{j}\right)$ for some $i, j \geq n$.

If $i=n, e_{n}=d\left(f x_{n}, T x_{j}\right) \leq \beta_{n}$ for all $n \geq 0$.

If $i \neq n$, then

$$
\begin{aligned}
e_{n}=d\left(f x_{i}, T x_{j}\right) & =d\left(W\left(T x_{i-1}, f x_{i-1}, \alpha_{i-1}\right), T x_{j}\right) \\
& \leq \alpha_{i-1} d\left(T x_{i-1}, T x_{j}\right)+\left(1-\alpha_{i-1}\right) d\left(f x_{i-1}, T x_{j}\right)
\end{aligned}
$$

If $\alpha_{i-1}=1$, we get $e_{n}=d\left(T x_{i-1}, T x_{j}\right)$ which does not hold by case (i).

If $\alpha_{i-1} \in(0,1)$, from (2.1.8), we have

$$
e_{n} \leq \alpha_{i-1} e_{n}+\left(1-\alpha_{i-1}\right) d\left(f x_{i-1}, T x_{j}\right)
$$

which implies that

$$
e_{n} \leq d\left(f x_{i-1}, T x_{j}\right) \leq e_{n}
$$

and hence $e_{n}=d\left(f x_{i-1}, T x_{j}\right)$.

Repeating this procedure, we obtain $e_{n}=d\left(f x_{n}, T x_{j}\right) \leq \beta_{n}$ for all $n \geq 0$.

Hence, $e_{n} \leq \beta_{n}$ for all $n \geq 0$. 
Case (iii). $e_{n}=d\left(f x_{i}, f x_{j}\right)$ for some $j>i \geq n$.

Now,

$$
\begin{aligned}
e_{n} & =d\left(f x_{i}, f x_{j}\right)=d\left(f x_{i}, W\left(T x_{j-1}, f x_{j-1}, \alpha_{j-1}\right)\right) \\
& \leq \alpha_{j-1} d\left(f x_{i}, T x_{j-1}\right)+\left(1-\alpha_{j-1}\right) d\left(f x_{i}, f x_{j-1}\right) .
\end{aligned}
$$

If $\alpha_{i-1}=1$, we get $e_{n}=d\left(f x_{i}, T x_{j-1}\right)$ which is discussed in case (ii).

If $\alpha_{i-1} \in(0,1)$, we get $e_{n}=d\left(f x_{i}, f x_{j-1}\right)$ for all $n \geq 0$.

Repeating this procedure, we obtain $e_{n}=d\left(f x_{i}, f x_{i}\right)=0$, a contradiction.

From the above three cases, we have $e_{n} \leq \beta_{n}$ for all $n$.

Hence, $e_{n}=\beta_{n}$ for all $n \geq 0$.

Since $\left\{e_{n}\right\}$ is a decreasing sequence of non-negative reals, it converges to a limit $a$ (say). Suppose $a>0$.

Since $a \leq e_{n}$ for all $n, \varphi(a) \leq \varphi\left(a_{n}\right)$ for all $n$.

Now consider

$$
\begin{aligned}
d\left(f x_{n}, T x_{j}\right) & =d\left(W\left(T x_{n-1}, f x_{n-1}, \alpha_{n-1}\right), T x_{j}\right) \\
& \leq \alpha_{n-1} d\left(T x_{n-1}, T x_{j}\right)+\left(1-\alpha_{n-1}\right) d\left(f x_{n-1}, T x_{j}\right) \\
& \leq \alpha_{n-1}\left[d\left(f x_{n-1}, f x_{j}\right)-\varphi\left(d\left(f x_{n-1}, f x_{j}\right)\right)\right]+\left(1-\alpha_{n-1}\right) d\left(f x_{n-1}, T x_{j}\right) \\
& \leq \alpha_{n-1}\left[e_{n-1}-\varphi\left(e_{n-1}\right)\right]+\left(1-\alpha_{n-1}\right) e_{n-1}
\end{aligned}
$$

so that

$$
\beta_{n}=\operatorname{Sup}\left\{d\left(f x_{n}, T x_{j}\right): j \geq n\right\} \leq e_{n-1}-\alpha_{n-1} \varphi\left(e_{n-1}\right)
$$

and hence

$$
e_{n} \leq e_{n-1}-\alpha_{n-1} \varphi\left(e_{n-1}\right)
$$

This implies that

$$
\alpha_{n-1} \varphi\left(e_{n-1}\right) \leq e_{n-1}-e_{n} .
$$

Adding the first $N$ terms in both sides of (2.1.9), we get

$$
\sum_{n=1}^{N} \alpha_{n-1} \varphi\left(e_{n-1}\right) \leq e_{0}-e_{N} .
$$

But $\varphi(a) \leq \varphi\left(e_{n}\right)$ for all $n=0,1,2, \ldots$.

Hence,

$$
\varphi(a) \sum_{n=1}^{N} \alpha_{n-1} \leq e_{0}-e_{N} \leq e_{0} .
$$


Letting $N \rightarrow \infty$, in (2.1.10), we get a contradiction, since $\sum_{n=0}^{\infty} \alpha_{n}=\infty$.

Hence $a=0$.

From the definition of the sequence $\left\{e_{n}\right\}$, we have $\left\{f x_{n}\right\}$ and $\left\{T x_{n}\right\}$ are Cauchy sequences and that

$$
\lim _{n \rightarrow \infty} d\left(f x_{n}, T x_{n}\right)=0 .
$$

Since $f(X)$ is complete,

$$
\lim _{n \rightarrow \infty} f x_{n}=f u, \text { for some } u \in X .
$$

From (2.1.11) using (2.1.12), we get

$$
\lim _{n \rightarrow \infty} T x_{n}=f u
$$

Now consider,

$$
d\left(T x_{n}, T u\right) \leq d\left(f x_{n}, f u\right)-\varphi\left(d\left(f x_{n}, f u\right)\right)
$$

Letting $n \rightarrow \infty$, from (2.1.14) using (2.1.12), (2.1.13) and the continuity of $\varphi$, we get

$$
d(f u, T u) \leq d(f u, f u)-\varphi(d(f u, f u))=0 .
$$

Hence, $T u=f u=z$ (say).

Since the pair of maps $(f, T)$ is weakly compatible, we have $T f u=f T u$ and hence $T z=f z$.

Now we claim that $T z=z$. Suppose $T z \neq z$.

Consider

$$
\begin{aligned}
d(T z, z) & =d(T z, T u) \leq d(f z, f u)-\varphi(d(f z, f u)) \\
& =d(T z, z)-\varphi(d(T z, z)),
\end{aligned}
$$

a contradiction. Hence, $T z=z$.

Hence, $f z=T z=z$.

The uniqueness of $z$ follows from the inequality (2.1.1).

Hence, the theorem follows.

Corollary 2.2. Let $(X, d)$ be a complete convex metric space with convex structure $W$ and $T: X \rightarrow X$ be a selfmap. Assume that there exists a $\varphi \in \Phi$ such that

$$
d(T x, T y) \leq d(x, y)-\varphi(d(x, y))
$$

for all $x, y \in X$. Then, for $x_{0} \in X$, the modified Mann iteration $\left\{x_{n}\right\}$ defined by 


$$
x_{n+1}=W\left(T x_{n}, x_{n}, \alpha_{n}\right), n=0,1,2, \ldots,
$$

where $0<\alpha_{n} \leq 1$ for each $n$, is Cauchy provided $\sum_{n=0}^{\infty} \alpha_{n}=\infty$, and $\left\{x_{n}\right\}$ converges to $z$ (say) in $X$ and $z$ is the unique fixed point of $T$.

Proof. Follows from Theorem 2.1 by choosing $f$ to be the identity mapping on $X$.

The following is an example in support of Theorem 2.1.

Example 2.3. Let $X=(0,1)$ with the usual metric $d$. Let $I=[0,1]$. We define $W: X \times X \times I \rightarrow X$ by $W(x, y, \lambda)=\lambda x+(1-\lambda) y$ for all $x, y \in X$ and for all $\lambda \in I$. Then $W$ is a convex structure on $X$ so that $X$ is a convex metric space with convex structure $W$. We define mappings $T, f: X \rightarrow X$ by

$$
T x=\left\{\begin{array}{r}
\frac{1}{2} \quad \text { if } 0<x<\frac{2}{3} \\
1-\frac{1}{2} x \text { if } \frac{2}{3} \leq x<1
\end{array}\right.
$$

and

$$
f x=\left\{\begin{aligned}
\frac{1}{3} & \text { if } 0<x<\frac{2}{3} \\
\frac{4}{3}-x & \text { if } \frac{2}{3} \leq x<1
\end{aligned}\right.
$$

Here we observe that $f(X)=\left[\frac{1}{3}, \frac{2}{3}\right]$ is a convex and complete subset of $X$ and $T(X)=$ $\left[\frac{1}{2}, \frac{2}{3}\right] \subset f(X)$. Also the selfmaps $f$ and $T$ are weakly compatible on $X$ and satisfy the weakly contractive condition (2.1.1) with $\varphi: R_{+} \rightarrow R_{+}$defined by $\varphi(t)=\frac{1}{2} t$. Thus $f$ and $T$ satisfy all the hypotheses of Theorem 2.1 .

Next we show that for any $x_{0} \in X$, the modified Mann iteration defined by (1.9.1) converges to the unique common fixed point of $f$ and $T$, which is the conclusion of Theorem 2.1.

Let $x_{0} \in X$ and $0<\alpha_{j} \leq 1$ for all $j=0,1,2, \ldots$, with $\sum_{j=0}^{\infty} \alpha_{j}=\infty$.

We have the following two cases.

Case (i). $x_{0} \in\left(0, \frac{2}{3}\right)$.

In this case, $f x_{0}=\frac{1}{3}$ and $T x_{0}=\frac{1}{2}$. Now

$f x_{1}=W\left(T x_{0}, f x_{0}, \alpha_{0}\right)=\alpha_{0} T x_{0}+\left(1-\alpha_{0}\right) f x_{0}=\frac{2}{3}-\frac{1}{3}\left(1-\frac{1}{2} \alpha_{0}\right)$.

This implies that $f x_{1} \in\left(\frac{1}{3}, \frac{1}{2}\right]$ so that $\frac{2}{3}-\frac{1}{3}\left(1-\frac{1}{2} \alpha_{0}\right)=\frac{4}{3}-x_{1}$; and $x_{1}=\frac{2}{3}+\frac{1}{3}\left(1-\frac{1}{2} \alpha_{0}\right)<1$. Hence $T x_{1}=\frac{2}{3}-\frac{1}{6}\left(1-\frac{1}{2} \alpha_{0}\right)$. Again, $f x_{2}=W\left(T x_{1}, f x_{1}, \alpha_{1}\right)=\alpha_{1} T x_{1}+\left(1-\alpha_{1}\right) f x_{1}=\frac{2}{3}-\frac{1}{3}\left(1-\frac{1}{2} \alpha_{0}\right)\left(1-\frac{1}{2} \alpha_{1}\right)$.

On continuing this process, inductively we obtain a sequence $\left\{x_{n}\right\}$ in $X$ such that $f x_{n+1}=\frac{2}{3}-\frac{1}{3} \prod_{j=0}^{n}\left(1-\frac{1}{2} \alpha_{j}\right), n=0,1,2, \ldots$ Hence, $f x_{n} \rightarrow \frac{2}{3}$ as $n \rightarrow \infty$.

Case (ii). $x_{0} \in\left[\frac{2}{3}, 1\right)$. 
Now, $f x_{0}=\frac{4}{3}-x_{0}$ and $T x_{0}=1-\frac{1}{2} x_{0}$.

Again,

$$
f x_{1}=W\left(T x_{0}, f x_{0}, \alpha_{0}\right)=\alpha_{0} T x_{0}+\left(1-\alpha_{0}\right) f x_{0}=\frac{2}{3}-\left(x_{0}-\frac{2}{3}\right)\left(1-\frac{1}{2} \alpha_{0}\right) .
$$

This implies that $f x_{1} \in\left(\frac{1}{2}, \frac{2}{3}\right]$ so that $x_{1}=\frac{2}{3}-\left(x_{0}-\frac{2}{3}\right)\left(1-\frac{1}{2} \alpha_{0}\right)$ and hence $T x_{1}=$ $\frac{2}{3}-\frac{1}{2}\left(x_{0}-\frac{2}{3}\right)\left(1-\frac{1}{2} \alpha_{0}\right)$. Again,

$$
f x_{2}=W\left(T x_{1}, f x_{1}, \alpha_{1}\right)=\alpha_{1} T x_{1}+\left(1-\alpha_{1}\right) f x_{1}=\frac{2}{3}-\left(x_{0}-\frac{2}{3}\right)\left(1-\frac{1}{2} \alpha_{0}\right)\left(1-\frac{1}{2} \alpha_{1}\right) .
$$

On continuing this process, inductively we obtain a sequence $\left\{x_{n}\right\}$ in $X$ such that $f x_{n+1}=\frac{2}{3}-\left(x_{0}-\frac{2}{3}\right) \prod_{j=0}^{n}\left(1-\frac{1}{2} \alpha_{j}\right), n=0,1,2, \ldots$ Hence, $f x_{n} \rightarrow \frac{2}{3}$ as $n \rightarrow \infty$.

In both the cases, for any $x_{0} \in X$, the modified Mann iteration $\left\{f x_{n}\right\}$ converges to $\frac{2}{3}$ and $\frac{2}{3}$ is the unique common fixed point of $f$ and $T$.

We observe that $f$ and $T$ are not compatible on $X$; for, we choose $x_{n}=\frac{2}{3}+\frac{1}{n}, n=4,5, \ldots$. Then $f x_{n}=\frac{2}{3}-\frac{1}{n}$, and $T x_{n}=\frac{2}{3}-\frac{1}{2 n}, n=4,5, \ldots$. We note that $f x_{n} \rightarrow \frac{2}{3}$ as $n \rightarrow \infty$ and $T x_{n} \rightarrow \frac{2}{3}$ as $n \rightarrow \infty$. Now $f T x_{n}=\frac{1}{3}$ and $T f x_{n}=\frac{1}{2}$ for all $n=4,5, \ldots$ so that $\lim _{n \rightarrow \infty} d\left(f T x_{n}, T f x_{n}\right)=\frac{1}{6} \neq 0$.

\section{An invariant approximation result}

Definition 3.1. Let $(X, d)$ be a metric space and let $E$ be a subset of $X$.

The set $P_{E}(u)=\{x \in E: d(x, u)=\operatorname{dist}(u, E)\}$ is called the set of best approximants to $u$ in $X$ out of $E$, where

$$
\operatorname{dist}(u, E)=\inf \{d(y, u): y \in E\} .
$$

Theorem 3.2. Let $E$ be a non-empty compact and convex subset of a convex metric space $X$ and $T, f: E \rightarrow E$ be selfmaps such that $u \in F(T) \cap F(f)$ for some $u \in X$. Assume that $T(\partial E \cap E) \subset E$ and $f\left(P_{E}(u)\right)=P_{E}(u)$. Further assume that there exists a $\varphi \in \Phi$ and for each $x, y \in P_{E}(u) \cup\{u\}$,

$$
d(T x, T y) \leq\left\{\begin{array}{l}
d(f x, f u)-\varphi(d(f x, f u)) \text { if } y=u \\
d(f u, f y)-\varphi(d(f u, f y)) \text { if } x=u \\
d(f x, f y)-\varphi(d(f x, f y)) \text { if } x, y \in P_{E}(u) .
\end{array}\right.
$$

If the pair of maps $(T, f)$ is weakly compatible, then $P_{E}(u) \cap F(f) \cap F(T) \neq \emptyset$.

Proof. Since $E$ is compact, $P_{E}(u) \neq \emptyset$. Clearly $P_{E}(u)$ is closed and hence compact, being a closed subset of a compact set $E$, so that $f\left(P_{E}(u)\right)$ is compact. Hence, $f\left(P_{E}(u)\right)$ is complete. 
Now we claim that $P_{E}(u)$ is convex.

Let $x, y \in P_{E}(u)$ and $\lambda \in I=[0,1]$. Then $d(x, u)=\operatorname{dist}(u, E)=\operatorname{dist}(y, u)$. Since $E$ is convex, $W(x, y, \lambda) \in E$ and hence $d(W(x, y, \lambda), u) \geq \operatorname{dist}(u, E)$. But

$$
d(W(x, y, \lambda), u) \leq(1-\lambda) d(x, u)+\lambda d(y, u)=\operatorname{dist}(u, E) .
$$

This implies that $d(W(x, y, \lambda), u)=\operatorname{dist}(u, E)$ and hence $W(x, y, \lambda) \in P_{E}(u)$.

Hence, $P_{E}(u)$ is convex subset of $X$.

Now let $x \in P_{E}(u)$. From the definition of $W$ we get,

$$
d(W(x, u, \lambda), u) \leq(1-\lambda) d(x, u) .
$$

Clearly,

$$
d(u, x)=d(u, W(x, u, \lambda))+d(W(x, u, \lambda), x) .
$$

Now from (3.2.2) and (3.2.3) we get,

$$
\begin{aligned}
d(u, x) & =d(u, W(x, u, \lambda))+d(W(x, u, \lambda), x) \\
& \leq d(u, W(x, u, \lambda))+\lambda d(x, u) .
\end{aligned}
$$

This implies that

$$
(1-\lambda) d(x, u) \leq d(u, W(x, u, \lambda)) .
$$

From (3.2.2) and (3.2.4), we obtain

$$
d(W(x, u, \lambda), u)=(1-\lambda) d(x, u) .
$$

We observe from (3.2.5) that $W(x, u, 0)=x$ and $W(x, u, 1)=u$. Also, it follows from (3.2.5) that, if $\lambda=0$, then clearly $x \in \partial E \cap E$ and hence the set

$$
\{W(x, u, \lambda): 0<\lambda \leq 1\} \cap E=\emptyset .
$$

Hence, $x \in \partial E \cap E$ and hence $P_{E}(u) \subset \partial E \cap E$.

Thus, $T\left(P_{E}(u)\right) \subset T(\partial E \cap E) \subset E$.

Therefore, $T x \in E$ and also $f x \in P_{E}(u)$ for all $x \in P_{E}(u)$.

Now consider

$$
\begin{aligned}
d(T x, u) & =d(T x, T u) \leq d(f x, f u)-\varphi(d(f x, f u)) \\
& \leq d(f x, u) \\
& \leq d(u, E) .
\end{aligned}
$$

This implies that $d(T x, u) \leq \operatorname{dist}(u, E)$. But $T x \in E$. 
Hence, $d(T x, u)=\operatorname{dist}(u, E)$, that is, $T x \in P_{E}(u)$.

Therefore, $T\left(P_{E}(u)\right) \subset P_{E}(u)=f\left(P_{E}(u)\right)$.

Hence all the hypotheses of Theorem 2.1 are satisfied. Hence by Theorem 2.1, there exists $z \in P_{E}(u)$ such that $f z=T z=z$.

Hence, $P_{E}(u) \cap F(f) \cap F(T) \neq \emptyset$.

\section{References}

[1] Ya. I. Alber and S. Guerre-Delabriere, Principle of weakly contractive maps in Hilbert spaces, New Results in Operator Theory and its Applications, (Ed. by I. Gohberg and Yu. Lyubich), Oper. Theory Adv. Appl., Birkhauser, Basel, 98 (1997), 7-22.

[2] A. Azam and M. Shakeel, Weakly contractive maps and common fixed points, MATEMATNYKN BECHNK, 60 (2008), 101-106.

[3] I. Beg and M. Abbas, Coincidence points and invariant approximation for mappings satisfying generalized weak contractive condition, Fixed Point Theory and Applications, 2008, ID $74503,1-7$.

[4] G. Jungck, Compatible mappings and common fixed points "Revised", Internat. J. Math. Math. Sci., 17(1994), 37-40.

[5] G. Jungck and B. E. Rhoades, Fixed points for set valued functions with out continuity, Indian J. Pure. Appl. Math., 29(1998), 227-238.

[6] W.R. Mann, Mean value methods in iteration, Proc. Amer. Math. Soc., 4(1953), 506-510.

[7] B.E. Rhoades, Some theorems on weakly contractive maps, Nonlinear Analysis, 47(2001), 2683-2693.

[8] S.P. Singh and B.A. Meade, On common fixed point theorems, Bull. Austral. Math. Soc., 16 (1977), 49-53.

[9] W. Takahashi, A convexity in metric space and nonexpansive mappings, I. Kodai Math. Sem. Rep., 22 (1970), 142-149.

Department of Mathematics, Andhra University, Visakhapatnam - 530 003, India.

E-mail: gvr_babu@hotmail.com

Department of Mathematics, Andhra University, Visakhapatnam - 530 003, India.

Permanent address: Department of Mathematics, Jimma University, Jimma, P.O. Box 378, Ethiopia.

E-mail: alemg1972@gmail.com 